\title{
Growth of Metal Oxide Nanowires from Supercooled Liquid Nanodroplets
}

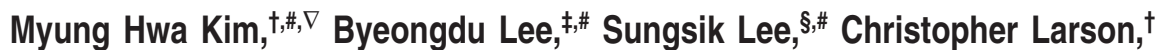 \\ Jeong Min Baik,,$+\circ$ Cafer T. Yavuz, ${ }^{\dagger}$ Sönke Seifert, ${ }^{\ddagger}$ Stefan Vajda, $,\|\|,, \perp$ \\ Randall E. Winans, ${ }^{\ddagger}$ Martin Moskovits, ${ }^{\dagger}$ Galen D. Stucky, ${ }^{*, \dagger}$ and Alec M. Wodtke ${ }^{\star, \dagger}$ \\ Department of Chemistry \& Biochemistry, University of California at Santa Barbara, \\ Santa Barbara, California 93106, X-ray Science Division, Advanced Photon Source, \\ Argonne National Laboratory, 9700 South Cass Avenue, Argonne, Illinois 60439, \\ Chemical Science and Engineering Division, Argonne National Laboratory, 9700 \\ South Cass Avenue, Argonne, Illinois 60439, Center for Nanoscale Materials, Argonne \\ National Laboratory, 9700 South, Cass Avenue, Argonne, Illinois 60439, and \\ Department of Chemical Engineering, School of Engineering and Applied Science, \\ Yale University, 9 Hillhouse Avenue, New Haven, Connecticut 06520
}

Received July 22, 2009; Revised Manuscript Received September 1, 2009

\begin{abstract}
Nanometer-sized liquid droplets formed at temperatures below the bulk melting point become supercooled as they grow through Ostwald ripening or coalescence and can be exploited to grow nanowires without any catalyst. We used this simple approach to synthesize a number of highly crystalline metal oxide nanowires in a chemical or physical vapor deposition apparatus. Examples of nanowires made in this way include $\mathrm{VO}_{2}, \mathrm{~V}_{2} \mathrm{O}_{5}, \mathrm{RuO}_{2}, \mathrm{MoO}_{2}, \mathrm{MoO}_{3}$, and $\mathrm{Fe}_{3} \mathrm{O}_{4}$, some of which have not been previously reported. Direct evidence of this new mechanism of nanowire growth is found from in situ 2-dimensional GISAXS (grazing incidence small angle X-ray scattering) measurements of $\mathrm{VO}_{2}$ nanowire growth, which provides quantitative information on the shapes and sizes of growing nanowires as well as direct evidence of the presence of supercooled liquid droplets. We observe dramatic changes in nanowire growth by varying the choice of substrate, reflecting the influence of wetting forces on the supercooled nanodroplet shape and mobility as well as substrate-nanowire lattice matching on the definition of nanowire orientation. Surfaces with defects can also be used to pattern the growth of the nanowires. The simplicity of this synthesis concept suggests it may be rather general in its application.
\end{abstract}

Understanding nanowire growth has the potential to improve our ability to fabricate devices such as gas sensors, photovoltaic cells, batteries, photonic crystals, and photocatalytic cells ${ }^{1-4}$ as well as providing important knowledge about the physical and chemical events that characterize anisotropic crystal formation.

Several methods for fabricating nanowires have been reported including liquid phase self-assembly and templated growth in nanometer sized pores. ${ }^{5}$ One of the most commonly used methods involves chemical vapor deposition

\footnotetext{
* To whom correspondence should be addressed. E-mail: stucky@ chem.ucsb.edu; wodtke@chem.ucsb.edu.

${ }^{\dagger}$ University of California at Santa Barbara.

* Advanced Photon Source, Argonne National Laboratory.

${ }^{\S}$ Chemical Science and Engineering Division, Argonne National Laboratory.

"Center for Nanoscale Materials, Argonne National Laboratory.

${ }^{\perp}$ Yale University.

\# These authors contributed equally to this work.

${ }^{\nabla}$ Current Address: Department of Chemistry and Nano Science, Ewha Womans University, Seoul, 120-750 Korea.

${ }^{\circ}$ Current Address: School of Advanced Materials and Systems Engineering, Kumoh National Institute of Technology, Gumi Gyungbuk, 730-701 Korea.
}

(CVD) or physical vapor transport. Here, a solid sample may be heated in a tube furnace in vacuum or in a streaming gas to produce a gas-phase precursor, which results in crystalline nanowire growth in the downstream region. The mechanism of growth may resemble sublimation followed by recrystallization, a process referred to as vapor-solid (VS) growth. ${ }^{6,7}$ Alternatively, nanometer sized metal particles can be used to catalyze nanowire growth. In this case, the metal particle may be molten, forming a eutectic solution with the precursor. As precursor material adsorbs and dissolves into the metal catalyst, solid nanowire crystallizes out as the solution becomes saturated. This growth mechanism is referred to as vapor-liquid-solid (VLS) $)^{8,9}$ or "metal catalytic" growth. Recently, it was also proposed that solid catalyst particles can be used to catalyze nanowire growth by a vapor-solid-solid (VSS) mechanism. ${ }^{10,11}$ Both VLS and VSS require catalysts to grow nanowires from them.

In this letter we describe an alternative platform for metal oxide nanowire growth without use of a catalyst exploiting the kinetics of nanometer scale liquid droplet growth. While nanowire growth without catalysts is not new, this work 
shows clear evidence of a remarkably simple physical principle, Ostwald ripening to produce supercooled liquid nanodroplets, that is quite useful for exploring nanowire growth.

It is well-known that as bulk solids are reduced to sizes smaller than about $100 \mathrm{~nm}$, melting point reduction occurs and follows the Gibbs-Thomson equation

$$
\Delta T_{\mathrm{M}}=T_{\mathrm{M}}-T_{\mathrm{M}}(\infty)=\frac{4 \sigma T_{\mathrm{M}}}{d \Delta H_{\mathrm{F}} \rho_{\mathrm{S}}}
$$

where $T_{\mathrm{M}}$ is the reduced melting temperature (the bulk melting temperature, $\left.T_{\mathrm{M}}(\infty)\right)$, $\rho_{\mathrm{S}}$ is the density of the solid, $\Delta H_{\mathrm{F}}$ is the latent heat of fusion, $d$ is the particle diameter, and $\sigma$ is the liquid-solid interface energy, respectively. ${ }^{13-16}$

Using electron diffraction methods, melting at temperatures as low as $477{ }^{\circ} \mathrm{C}$ has been reported for nanometer sized $\mathrm{Au}$ particles. By comparison the bulk melting point of $\mathrm{Au}, T_{\mathrm{m}, \mathrm{Au}}$, is $1064{ }^{\circ} \mathrm{C} .{ }^{13} \mathrm{X}$-ray diffraction has shown $\mathrm{Pb}$ particles melting at temperatures as low as $202^{\circ} \mathrm{C}\left(T_{\mathrm{m}, \mathrm{Pb}}=377^{\circ} \mathrm{C}\right) .{ }^{16}$ Thus, under nanowire growth conditions below the bulk melting point of the precursor, condensed precursors on a substrate must not by necessity form a crystal (or for that matter a solid) but may rather form a liquid nanodroplet. Of course, formation of liquid nanodroplets on various solid substrates at elevated temperatures below the bulk melting point may also lead to rapid droplet growth through Ostwald ripening ${ }^{17}$ or coalescence of small droplets. Naturally, the growing nanodroplets must then become supercooled as their melting points rise with increasing size. It is then logical to postulate that these supercooled nanodroplets might be useful nucleation sites for nanowire growth. In contrast to VLS and VSS growth, which requires catalysts and carefully controlled conditions which exploit the thermodynamics properties of eutectic mixtures, this growth mechanism might be more generally applicable, even when useful eutectics cannot be found or do not exist.

The limited means of obtaining high spatial-resolution in situ structural information on nanowire growth remains a challenge when studying the proposed growth mechanism. Real time transmission electron microscopy (RTTEM) is one of the most powerful approaches to this problem. For example, RTTEM studies of $\mathrm{GaN}$ nanowire growth from $\mathrm{Ga}$ particles and Ge nanowire growth from Au particles helped reveal essential aspects of the VSS and VLS mechanisms. ${ }^{10,18,19}$ Unfortunately, this technique cannot be applied under realistic reaction environments like atmospheric pressure condition. On the other hand, grazing incidence small angle X-ray scattering (GISAXS) offers a complementary approach to in situ monitoring of nanometer-scale growth. This method provides real-time, nanometer-resolution spatial information and can easily be applied under a wide range of temperatures and pressures with essentially any growthsubstrate. ${ }^{20-23}$ One study has been reported using GISAXS to observe in situ nanoparticle growth $;{ }^{20}$ however, nanowire growth has yet to be explored with this technique. Like many other technique in situ X-ray scattering techniques are usually not used to follow the growth of a single nanowire but rather they can be used to observe structural changes over a wide area where many different objects, such as nanodroplet, nanocrystal and nanowire, exist at a same time. Their relative amounts and sizes over the growth time can be determined from the characteristic scattering features of each object. Our growth motif suggests that the formation of liquid nanodroplets should be observed at the early stage of in situ growth measurements and its number will be reduced as coverage of nanowires on a substrate is increased. Nanocrystals that are nucleated from bigger saturated droplets will be formed not only at the early stages of deposition but also at later stages as long as there are enough nanodroplets to coalescence. Growth of nanowires must not be limited to its length direction, but may grow in other directions but with much slower speed.

In this paper, we show direct observations of nanometer sized liquid droplets of vanadium oxide well below its bulk melting point, formation of nanocrystals and growth of nanowires using in situ GISAXS as well as SEM (scanning electron microscopy) for quenched samples. Using the mechanistic insights derived from these studies, we have successfully produced single crystal nanowires from a variety of metal oxides including: $\mathrm{VO}_{2}, \mathrm{~V}_{2} \mathrm{O}_{5}, \mathrm{RuO}_{2}, \mathrm{MoO}_{2}, \mathrm{MoO}_{3}$, and $\mathrm{Fe}_{3} \mathrm{O}_{4}$, some of which have not been previously reported. Important aspects of this synthesis platform include: a remarkable sensitivity of the growth to choice of substrate, the possibility of patterning growth through substrate defects, highly crystalline nanowire growth and a relatively wide range of usefulness for different materials.

$\mathrm{VO}_{2}$ nanowire growth experiments ${ }^{24}$ were carried out in a homemade portable cubic CVD chamber equipped with a boron nitride (BN) heater and two window ports transparent to the X-ray beam at the 12ID-C beamline of the Advanced Photon Source at Argonne National Laboratory. To study the isothermal growth of nanowires at different temperatures, $6.0 \mathrm{mg}$ of $\mathrm{VO}_{2}$ fine meshed powder (99.9\%, Aldrich) without further purification was placed at one end of a single crystal $\mathrm{Si}(100)$ substrate, which was covered by a $200 \mathrm{~nm}$ thick thermally grown $\mathrm{SiO}_{2}$ layer. The CVD chamber was initially flushed by continuously flowing high purity $\mathrm{He}(99.999 \%)$ carrier gas with a flow rate of $200 \mathrm{sccm}$ (standard centimetercubic per minute) under atmospheric pressure and then heated to selected temperatures in the range of $450-650{ }^{\circ} \mathrm{C}$ within $5 \mathrm{~min}$ and held at a constant temperature for 10-30 $\mathrm{min}$ with constant He flow. For the in situ GISAXS experiments, a narrow and well-collimated incident $8 \mathrm{keV} \mathrm{X}$-ray beam was directed toward the $\mathrm{SiO}_{2} / \mathrm{Si}$ surface, $4 \mathrm{~mm}$ away from the $\mathrm{VO}_{2}$ powder source, at a grazing angle, $0.15^{\circ}$, which is below the critical angle of $\mathrm{SiO}_{2} / \mathrm{Si}(100)$ substrate leading to total external reflection. The GISAXS data were then collected on a $2048 \times 2048$-pixel two-dimensional CCD camera located about $2 \mathrm{~m}$ downstream from the sample. GISAXS images were recorded every $20 \mathrm{~s}$ with $1 \mathrm{~s}$ irradiation time. After a well-defined reaction time of $30 \mathrm{~min}$, the $\mathrm{SiO}_{2} / \mathrm{Si}$ substrate was quickly cooled to room temperature. The surface morphology, structure, and elemental analysis of the as-grown samples were subsequently examined using scanning electron microscopy (SEM), X-ray 


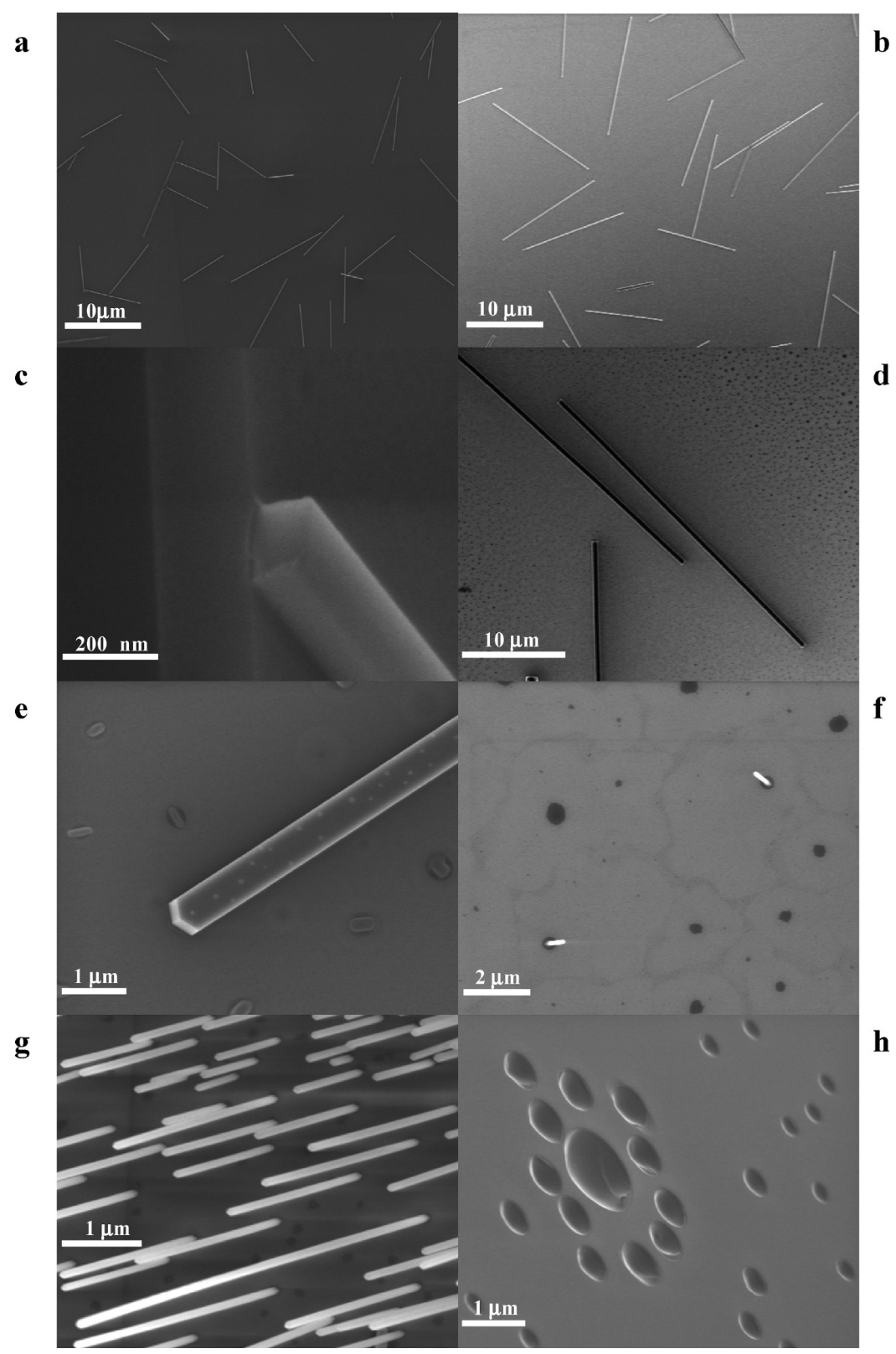

Figure 1. Scanning electron micrographs of vanadium oxide growth structures resulting from $\mathrm{CVD}$. (a) $\mathrm{VO}_{2}$ nanowires grown on a $\mathrm{SiO} / 2 / \mathrm{Si}$ substrate at $550{ }^{\circ} \mathrm{C}$ using $\mathrm{VO}_{2}$ as a precursor in flowing $\mathrm{He}$ for $30 \mathrm{~min}$. (b) Same as in panel a, but the growth temperature was $600{ }^{\circ} \mathrm{C}$. (c) A cross-sectional view of $\mathrm{VO}_{2}$ nanowire grown as in panel a shown in high magnification. A rectangular cross-section is easily observed. (d) $\mathrm{VO}_{2}$ nanowire growing in plane at $600{ }^{\circ} \mathrm{C}$ in the presence of nanodroplets. The lower density of nanodroplets in the vicinity of the nanowires is taken as evidence that the nanodroplets feed material to the growing nanowires. (e) Droplet-like structures feeding material to the $\mathrm{VO}_{2}$ nanowire growth at $600{ }^{\circ} \mathrm{C}$ on $\mathrm{SiO}_{2} / \mathrm{Si}$. (f) $\mathrm{VO}_{2}$ nanowires growing directly from droplet-like structures at $550{ }^{\circ} \mathrm{C}$ on $\mathrm{SiO} / 2 / \mathrm{Si}$. (g) $\mathrm{VO}_{2}$ nanowires on $\mathrm{r}$-cut sapphire grown at $650{ }^{\circ} \mathrm{C}$ in $\mathrm{He} . \mathrm{VO}_{2}$ nanowires form an angle of $60^{\circ}$ with respect to the basal $\mathrm{r}$-cut sapphire and appear to grow from droplet like structures. (h) A particularly clear image of droplet-like structures under a tilted angle.

diffraction (XRD), micro Raman spectroscopy, and atomic force microscopy (AFM). For other metal oxide systems, nanowires were grown by atmospheric pressure physical vapor deposition (APPVD) using a quartz tube furnace with flowing Ar or He carrier gas.

Vanadium oxide is an ideal system to demonstrate the growth motif outlined above. Only two oxides of vanadium are important under our conditions of temperature and $\mathrm{O}_{2}$ partial pressure: a low-melting oxide, vanadium(V) oxide, $\mathrm{V}_{2} \mathrm{O}_{5},\left(T_{\mathrm{M}, \mathrm{V} 2 \mathrm{O} 5}=690{ }^{\circ} \mathrm{C}\right)$, and a high melting oxide, vanadium(IV) oxide, $\mathrm{VO}_{2},\left(T_{\mathrm{M}, \mathrm{VO} 2}=1967{ }^{\circ} \mathrm{C}\right)$. Vanadium metal has a melting point of $1900{ }^{\circ} \mathrm{C}$ far above the temperatures of this work. Under mildly reducing conditions (flowing $\mathrm{He}$ ) at temperatures between 550 and $650{ }^{\circ} \mathrm{C}$, supercooled liquid $\mathrm{V}_{2} \mathrm{O}_{5}$ nanodroplets formed by vapor transport from solid $\mathrm{VO}_{2}$ become sites for $\mathrm{VO}_{2}$ crystal growth. Alternatively, at lower temperatures $\left(450-500{ }^{\circ} \mathrm{C}\right)$ or under mildly oxidizing conditions, where $1 \mathrm{sccm}$ of $\mathrm{O}_{2}$ is mixed into the rare gas flow, $\mathrm{V}_{2} \mathrm{O}_{5}$ nanowires can be formed, using $\mathrm{V}_{2} \mathrm{O}_{5}$ powder as a precursor.

Initial scanning electron microscopy (SEM) imaging of the post reaction samples show evidence of nanowire growth and liquid droplet formation. Figure 1, panels a and b, shows SEM images of $\mathrm{VO}_{2}$ nanowires grown at 550 and $600{ }^{\circ} \mathrm{C}$, 
respectively, under the conditions just described. SEM data show that the nanowires are randomly oriented in the plane of substrate and that the dimension of the nanowires depends on their growth temperature and time. X-ray diffraction patterns (XRD) of these nanowires (Figure S1) confirms the presence of highly crystalline $\mathrm{VO}_{2}$ structures, exhibiting two indexed peaks, (011) and (022), consistent with the monoclinic $\mathrm{VO}_{2}$ structure. The high magnification SEM image (Figure 1c) reveals that the $\mathrm{VO}_{2}$ nanowires possess a rectangular cross-section, similar to previous reports for nanowires grown by vapor transport at higher temperature and lower pressure. ${ }^{25,26}$ Figure 1d shows nanowires with $\sim 100 \mathrm{~nm}$ width more than $30 \mu \mathrm{m}$ in length, in the presence of many droplet-like structures that are $\sim 50 \mathrm{~nm}$ in size. The droplet density is dramatically depleted within about $2 \mu \mathrm{m}$ of the nanowires, suggesting that the nanodroplets are being consumed by the nanowires during growth.

These droplets appear in all of the images of Figure 1 and range in size from $\sim 50 \mathrm{~nm}$ to $\sim 2 \mu \mathrm{m}$. In three of the images (especially panels $\mathrm{e}-\mathrm{g}$ ), one can see what appear to be nanowires growing directly out of droplets. Figure 1e also shows the presence of $\sim 50 \mathrm{~nm}$ droplet-like structures on the faces of the nanowire themselves, consistent with the idea of nanodroplets feeding the growth of the nanowires. Additional SEM images showing nanowire growth from droplets are shown in Figure S2 a-d. By adjusting the growth temperature, $\mathrm{O}_{2}$ partial pressure and choice of substrate, inplane growth (Figure 1d) or out-of-plane growth (Figure 1, panels $\mathrm{f}$ and $\mathrm{g}$ ) can be induced and nanowire composition $\left(\mathrm{VO}_{2}\right.$ vs $\mathrm{V}_{2} \mathrm{O}_{5}$, Figure $\mathrm{S} 2 \mathrm{e}$ and f) can be influenced. Figure $1 \mathrm{~h}$ shows a clear image of droplet-like structures in the late stages of aggregation and growth.

In addition to XRD data on nanowires, evidence regarding the chemical composition of the nanowires and the dropletlike structures was obtained from Raman microscopy (Figure 2a). While substrate features dominate the spectrum, the Raman spectra of single nanowires exhibit two characteristic sharp peaks at 191 and $223 \mathrm{~cm}^{-1}$ and a broad peak at 390 $\mathrm{cm}^{-1}$, which are attributable to single crystal monoclinic $\mathrm{VO}_{2}{ }^{27,28}$ In contrast to nanowires, the Raman spectra of droplet-like structures (like Figure 1h) consistently show features at 148, 264, and $700 \mathrm{~cm}^{-1}$, which are characteristic of crystalline or amorphous $\mathrm{V}_{2} \mathrm{O}_{5} \cdot{ }^{28}$

SEM imaging on postreaction samples in Figure $2 b$ provide snapshots of the nucleation within the saturated droplet and temperature dependent histograms of the size distribution of the nanocrystals. At low temperatures (550 $\left.{ }^{\circ} \mathrm{C}\right)$ this distribution is remarkably narrow and centered at about $110 \mathrm{~nm}$. At higher temperature $\left(650^{\circ} \mathrm{C}\right)$, the distribution becomes significantly broader and shifts to a larger average size. Larger crystal size at higher temperature is due to higher crystallization temperature; the size is inversely proportional to the degree of supercooling as seen in eq 1. It may be due to the larger size of saturated droplet at higher temperature since at lower growth temperatures, nanodroplets become supercooled at smaller sizes and tend to freeze sooner, whereas at higher temperatures, closer to the bulk melting point, nanodroplets grow to larger sizes before
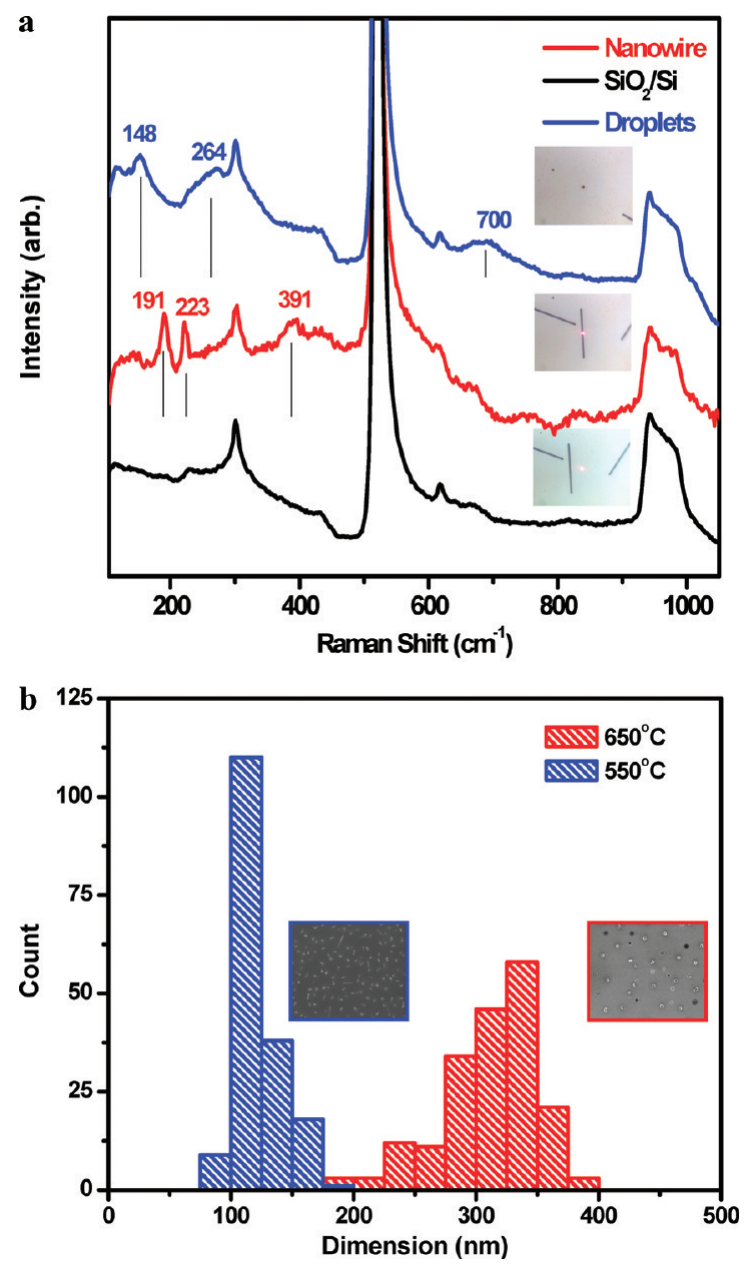

Figure 2. Raman spectra and particle size distributions of $\mathrm{VO}_{2}$ nanostructures. (a) Raman spectra of a single nanowire (red line) and droplet-like structures (blue line) on a $\mathrm{SiO}_{2} / \mathrm{Si}$ substrate (black line) after in situ experiment grown at $600{ }^{\circ} \mathrm{C}$ using $632.8 \mathrm{~nm}$ laser light with $0.57 \mathrm{~mW}$ power. Inset shows optical images with tightly focused laser beam spots (about $1 \mu \mathrm{m}^{2}$ ) on a droplet (top) like Figure $1 \mathrm{~h}$, on a nanowire (middle) and on the substrate (bottom). The 191, 222 , and $390 \mathrm{~cm}^{-1}$ peaks in the nanowire spectrum are characteristic Raman modes for monoclinic $\mathrm{VO}_{2}$. The 148,264 , and $700 \mathrm{~cm}^{-1}$ features in the droplet spectrum are attributed to $\mathrm{V}_{2} \mathrm{O}_{5}$ phase. (b) A histogram of the size distribution of crystalline structures at the early stage of growth from the droplet-like structures for 550 and $650{ }^{\circ} \mathrm{C}$. SEM images as insets show crystalline structures formed from the droplet-like structures.

solidification. At higher temperature diffusion causing Ostwald-ripening is also faster.

SEM imaging has inherent limits of spatial resolution rendering invisible droplets smaller than $\sim 30 \mathrm{~nm}$. Moreover, we can only use SEM data on postreaction products, and can only infer what conditions were present during the reaction. Despite this, the SEM imaging and the interpretation given above, suggest that smaller liquid droplets are present at an early stage of the nanowire growth. In situ GISAXS on the other hand provides a sequence of events in the formation of different objects and their growth. We first describe characteristic scattering features of three objects; nanodroplets, nanocrystals, and nanowires. We then present quantitatively analyzed GISAXS results. Note that GISAXS 

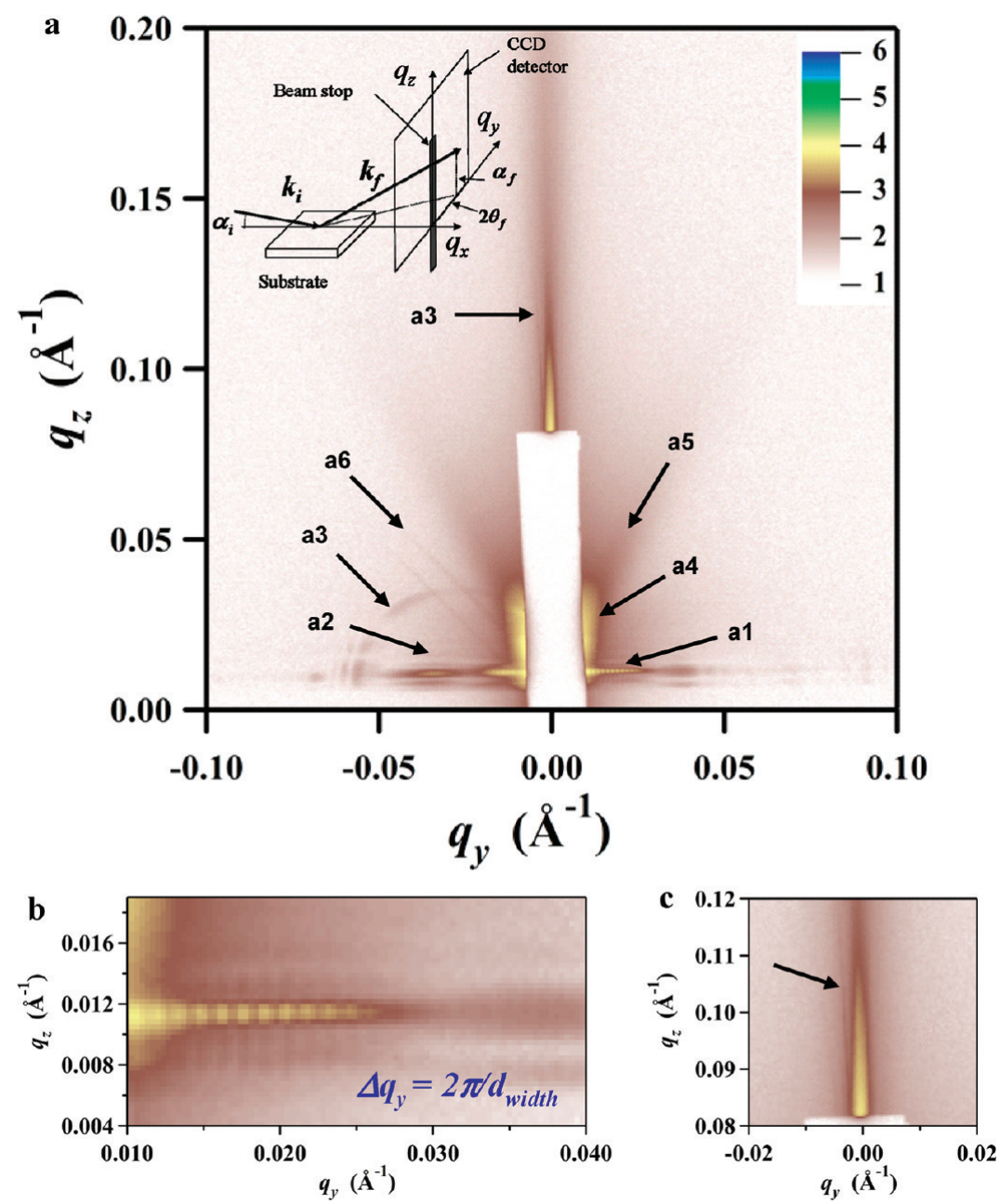

Figure 3. Experimental GISAXS image obtained during the $\mathrm{VO}_{2}$ nanowire growth. (a) A typical GISAXS image acquired at time $t=$ $1345 \mathrm{~s}$ at $600{ }^{\circ} \mathrm{C}$, showing distinctive features $(\mathrm{a} 1-\mathrm{a} 6)$ associated with nanowire as well as nanodroplets or nanocrystals. The inset shows a schematic diagram of the experimental setup. The incident and scattered X-ray beams are characterized by wave vectors $\mathbf{k}_{\mathrm{i}}$ and $\mathbf{k}_{\mathrm{f}}$ defined by the in-plane and out-of-plane angles, $2 \theta_{\mathrm{f}}, \alpha_{\mathrm{i}}$, and $\alpha_{\mathrm{f}}$, respectively. Scattering vectors $q$ are calculated from $(4 \pi / \lambda)$ sin $\theta_{\mathrm{f}}$, where $\theta_{\mathrm{f}}$ is scattering half angle and $\lambda$ is the wavelength of X-rays. (b) The high magnification image of the well-defined fringing pattern along the horizontal direction (a1), indicating that the width of nanowires could be calculated using the Bragg equation, $2 \pi / \Delta q_{y}$, where $\Delta q_{y}$ is defined as the frequency of the fringe. (c) The high magnification image of the characteristic "ring scattering" feature (a3) of nanowires oriented with respect to the X-ray beam direction.

structures larger than about $50 \mathrm{~nm}$ cannot be resolved in our setup unless they are highly monodisperse in size.

Figure 3a shows a typical GISAXS scattering pattern obtained during in situ $\mathrm{VO}_{2}$ nanowire growth at $600{ }^{\circ} \mathrm{C}$, under the same conditions as those used to generate the SEM images in Figure 1, panels b and d, where in-plane nanowire growth dominates. The 2-D GISAXS image represents several unique features originating from nanowires as well as nanodroplets or nanocrystals. First, the most distinct features observed are related to the well-defined fringe patterns (a1 and a2) and concentric rings (a3), respectively. As magnified in Figure 3b, vertical fringes (a1) with equal spacing along the horizontal axis $\left(q_{y}\right)$ can be identified with interferences between X-ray scattering beams from the two sides of a rectangular shaped nanowires that are aligned nearly parallel to the X-ray beam, corresponding to the width of the nanowire's rectangular cross-section (Figure 1c). The statistical average of the width of the nanowires is thus inversely proportional to the spacing between the fringe patterns. On the other hand, horizontal fringes (a2) along the vertical axis $\left(\mathrm{q}_{z}\right)$ originate from interferences between two scattering X-ray beams, which are reflected from the top of the nanowires and the surface of the substrate. In the same manner, the fringe spacing is inversely proportional to the height of the nanowires. Furthermore, these features can be clearly reproduced by simulations of GISAXS images for a nanowire with a rectangular cross-section as a function of the in-plane angle $\Phi$ between the nanowire and X-ray beam direction, using realistic experimental parameters (see Figure S3). Another intriguing feature of in situ nanowire GISAXS is the observation of concentric rings (a3) of varying radius (see Figure 3, panels a and c). These ring features also result from a nanowire that is slightly titled from the X-ray propagation direction as can be seen from simulations with changing orientation angle, shown in Figure S3. It should be pointed out that the diameter of the rings is so extraordinarily sensitive to the orientation angle, $\Phi$, of a nanowire with respect to the $\mathrm{X}$-ray beam direction that ring 


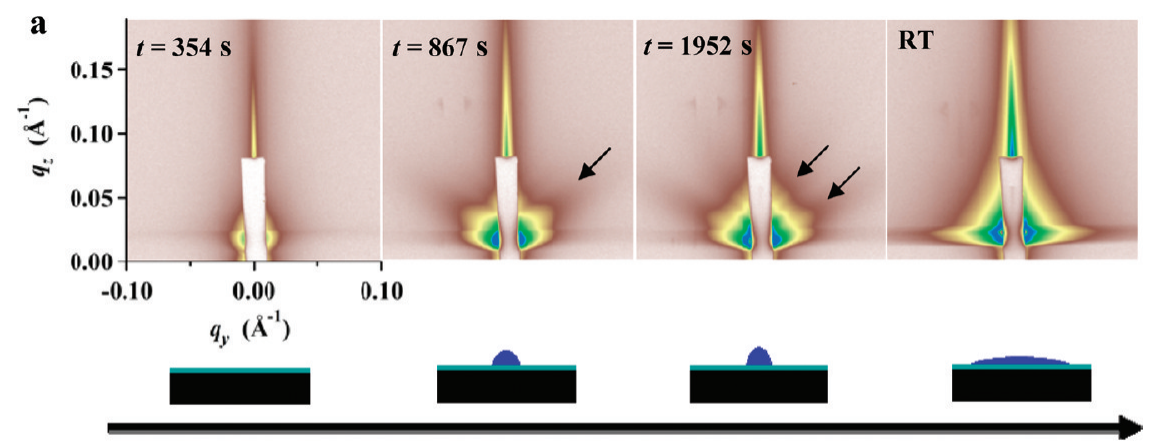

Time

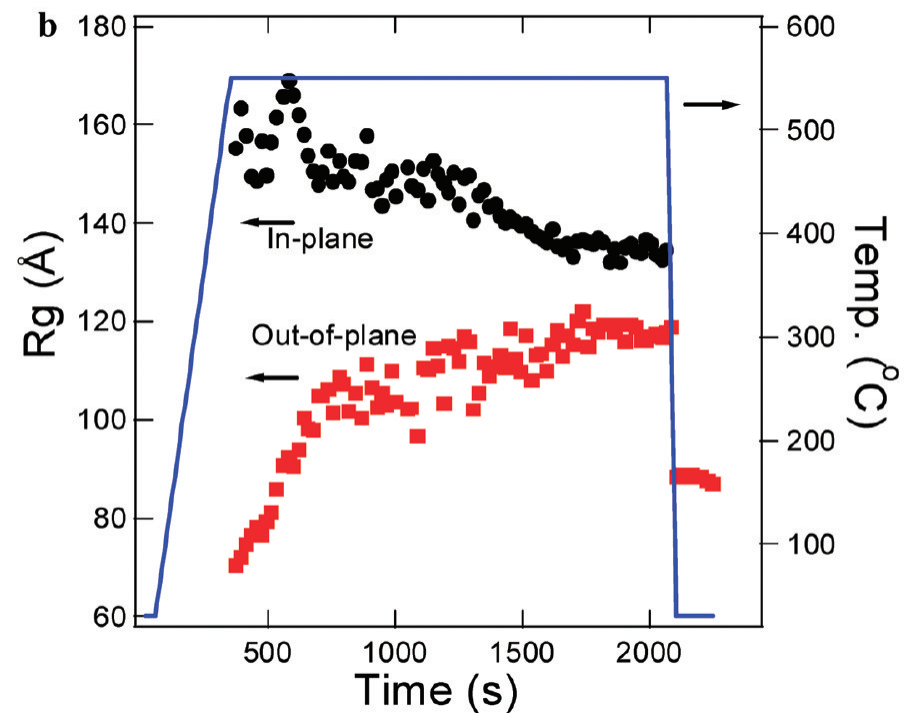

Figure 4. Real-time snapshot dynamics of the $\mathrm{VO}_{2}$ nanowire growth at $550{ }^{\circ} \mathrm{C}$. (a) Series of GISAXS images of VO $\mathrm{V}_{2}$ nanowire growth acquired at various times during in situ experiments at $550{ }^{\circ} \mathrm{C}$ under atmospheric pressure. The schematic diagrams represent the time profile of the shape of droplets extracted from horizontal and vertical cuts of these GISAXS patterns. Arrows point to the Bragg-rod scatterings originating from nanodroplets. (b) The time evolution of the height and width of droplets extracted from the form factor scattering component of panel a at $550{ }^{\circ} \mathrm{C}$. Notice that the red squares represent the height of nanodroplets, while the black circle represents the width. It should be also pointed out that after cooling, the width is beyond the measurement limit. The blue line shows the reactor temperature. Droplets initially wet the surface but become more spherical in shape with time and reach a steady state size. Upon temperature quenching at the end of the experiment a discontinuous reduction in height indicates a phase transition to solid.

shapes are already invisible for $\Phi>2.0^{\circ}$ (see Figure S3). Thus, the concentric ring patterns results from only a very small number of nanowires (or even a single nanowire). In fact, the one producing the concentric ring (a3) seen near the bottom of Figure 3a corresponds to a single nanowire with its long axis oriented $0.4^{\circ}$ away from the X-ray propagation direction. The period of the concentric ring is related to the length of the nanowire. In the image there are other sets of concentric rings with different curvatures such as that in Figure $3 \mathrm{c}$ while they are barely distinguishable.

The existence of featureless diffuse scattering (a4) represents nanoparticles and nanodroplets or nanocrystals with a polydispersed size distribution. In addition, the rod like scattering features (a5 and a6), so-called the Braggrod, typically indicate the presence of flat surfaces normal to the scattering rod. The rod becomes sharper or narrower as the flat surface becomes larger. Thus a5 indicates nanocrystals, and a6 reflects a crystalline object with a flat facet larger than several $\mu \mathrm{m}$. We have seen Braggrods like a6 from nanowires grown vertically with an angle. As seen from SEM image, however, most nanowires grow horizontally at $600{ }^{\circ} \mathrm{C}$.

Figure 4 a shows a series of in situ GISAXS images obtained as a function of time at $550{ }^{\circ} \mathrm{C}$. The time evolution of these images contains important information about the early stage growth dynamics and the nucleation of nanodroplets, which plays a crucial role in growing $\mathrm{VO}_{2}$ nanowires. Initially ( $t=354 \mathrm{~s}$ ), the image shows only background scattering from the $\mathrm{SiO}_{2} / \mathrm{Si}$ substrate. As time evolves, diffuse scattering is clearly observed and gradually develops as shown in Figure 4a $(t=867$ and $1952 \mathrm{~s})$, indicating that nanodroplets and nanocrystals are forming. Intensity of the diffuse scattering continuously increases indicative of an increase in the number of nanodroplets that are smaller than $50 \mathrm{~nm}$. Since GISAXS is not sensitive to polydisperse droplets larger than $50 \mathrm{~nm}$, it is hard to get information on larger saturated droplets. The broad Bragg-rod scattering denoted by arrows, however, indicates the formation of nanocrystals. In fact, the increase of the diffuse scattering intensity over time suggests that the growth or formation of 

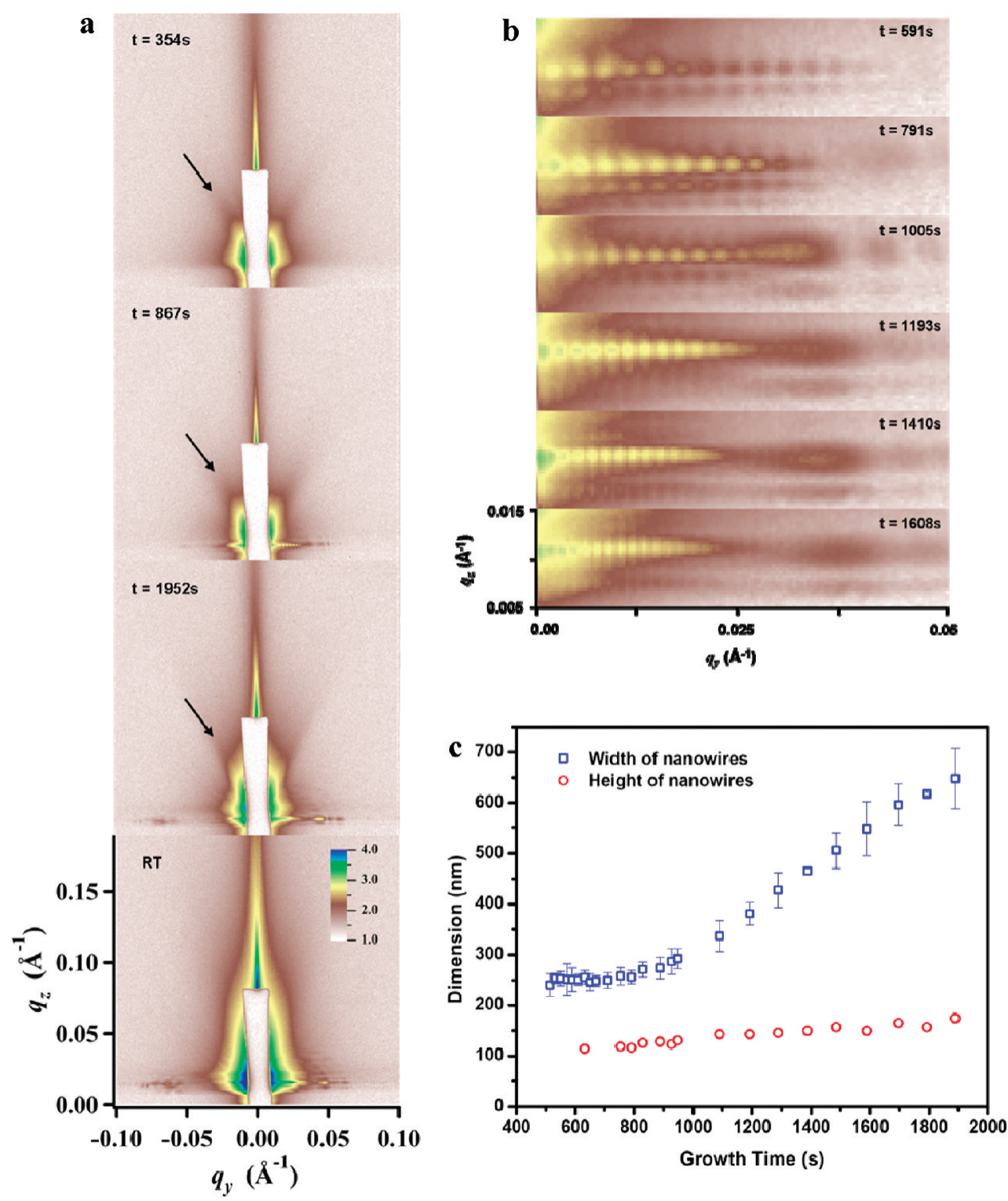

Figure 5. Real-time snapshot dynamics of the $\mathrm{VO}_{2}$ nanowire growth at $600{ }^{\circ} \mathrm{C}$. (a) A series of GISAXS images of $\mathrm{VO}_{2}$ nanowire growth acquired at various times during in situ experiments at $600{ }^{\circ} \mathrm{C}$ under atmospheric pressure. Arrows represent the Bragg-rod scatterings originating from nanodroplets. (b) In situ GISAXS fringe patterns of the nanowire growth measured at different growth times for $600{ }^{\circ} \mathrm{C}$, showing that the spacing is gradually narrower with growth time. Note that the average width of nanowires is $2 \pi / \Delta q$. (c) The growth time dependence of the average width and height for nanowires extracted from the analysis of fringe patterns for $600{ }^{\circ} \mathrm{C}$ like those shown in panel c.

nanodroplets is faster than its consumption to form nanocrystals or nanowires at this temperature.

By quantitatively analyzing horizontal and vertical cuts of these GISAXS patterns, we extract the sizes and shapes and how they are changing as a function of time. This is illustrated in Figure 4b. Here the black circles represent the in-plane dimension, width, of the nanodroplets while the red squares indicate the out-of-plane dimension, height. The blue line indicates the in situ growth temperature. One can clearly see that the width of the nanodroplets is relatively static during the growth period with a size of about $14 \mathrm{~nm}$, far smaller than all of the nanowires that are eventually formed. In contrast the height increases steeply and then more gradually reaches $12 \mathrm{~nm}$. Interesting changes on the scattering feature is found when the sample is cooled at the end of the growth process (see Figure $4 \mathrm{~b}, t=2200 \mathrm{~s}$ ). The remarkably sudden drop in the height, and increase in the width (width after the quench is not included in the Figure $4 \mathrm{~b}$ because it is far above the measurement limit, size $>100$ $\mathrm{nm}$ in this work) of the nanodroplets caused by the temperature drop indicates that under these conditions the droplets are in a highly mobile phase. Indeed, the sudden large volume increase can only be rationalized in terms of the coalescence of many nearby objects. This observation is direct evidence that these $20-30 \mathrm{~nm}$ nanodroplets are indeed liquid, as nanostructures in the solid state cannot change their shapes in such a dramatic fashion. This interpretation of the observations is also consistent with the results of Raman spectroscopy (Figure 2a) and SEM images (Figure 1, panels $\mathrm{f}$ and $\mathrm{h}$ ) discussed above, which show bigger droplet-like 
Table 1. The Nanowire Growth of Various Metal Oxides from Supercooled Liquid Nanodroplets

\begin{tabular}{|c|c|c|c|c|c|c|c|c|}
\hline $\begin{array}{c}\text { Nanowire } \\
\text { target }\end{array}$ & $\mathrm{VO}_{2}$ & $\mathrm{~V}_{2} \mathrm{O}_{5}$ & $\mathrm{MoO}_{2}$ & $\mathrm{MoO}_{3}$ & $\mathrm{RuO}_{2}$ & $\mathrm{FeO}$ & $\mathrm{Fe}_{3} \mathrm{O}_{4}$ & $\mathrm{Fe}_{2} \mathrm{O}_{3}$ \\
\hline Source & $\mathrm{VO}_{2}$ & $\mathrm{~V}_{2} \mathrm{O}_{5}$ & $\mathrm{MoO}_{2}$ & $\mathrm{MoO}_{3}$ & $\mathrm{RuO}_{2}$ & & $\mathrm{FeO}$ & \\
\hline$T_{M}$ & 1967 & 690 & 1100 & 795 & 1200 & 1370 & & 1566 \\
\hline $\mathbf{T}_{\text {Growth }}$ & $550-650$ & $450-550$ & 850 & $500-600$ & $600-950$ & & $1000-1100$ & \\
\hline $\mathrm{O}_{2}(\mathrm{sccm})$ & & 1 & & & 10 & & & \\
\hline $\begin{array}{l}\text { Nano } \\
\text { wires }\end{array}$ & X & $x$ & $x$ & $X$ & $x$ & & $x$ & \\
\hline
\end{tabular}

nanostructures with $\mathrm{V}_{2} \mathrm{O}_{5}$ composition. Recall that $\mathrm{V}_{2} \mathrm{O}_{5}$ is the lowest melting form of vanadium in the presence of oxygen.

Real time GISAXS imaging (Figure 5a) at a growth temperature of $600{ }^{\circ} \mathrm{C}$ contain much more complicated features than those of $550^{\circ} \mathrm{C}$ as described in Figure 3a. Both nanocrystals and nanodroplet are already observed at the beginning of the growth (see the Bragg-rod and diffuse scatterings in Figure 5a; $t=354 \mathrm{~s}$ ), indicating that the growth rate is much faster than at $550^{\circ} \mathrm{C}$. At $t=867 \mathrm{~s}$, the beginning of nanowire formation is unambiguously identified by the appearance of fringe patterns along the $\mathrm{q}_{y}$ and $\mathrm{q}_{z}$ directions as well as by the appearance of concentric rings which correspond to nanowires aligned nearly parallel to the X-ray beam. In particular, the spacing of the fringe pattern along the $q_{y}\left(q_{z}\right)$ direction (see Figure 5b) gradually narrows as a function of time during the growth process, allowing in situ observation of the change of the average width (and height) of the nanowires. Figure $5 \mathrm{c}$ shows the time dependencies of the average width and height quantitatively derived from analysis of GISXAS images at $600{ }^{\circ} \mathrm{C}$. We have used AFM to test the reliability of this in situ analytic method by obtaining independently the final widths and heights of the as grown nanowires. See Figure S4, panels a and b, where one can quickly see that the agreement with the GISAXS analysis is excellent.

The intensities of the Bragg-rod and diffuse scattering from nanodroplets are relatively static with time in the $600{ }^{\circ} \mathrm{C}$ experiments in contrast to similar data obtained at $550{ }^{\circ} \mathrm{C}$. This implies that at $600{ }^{\circ} \mathrm{C}$ nanodroplets beyond a certain size are consumed by the growing nanowires. Furthermore, the attenuation of the growth of the liquid droplets occurs simultaneously with the increase in the contribution of the nanowire scattering features. Thus, the scattering images at $600{ }^{\circ} \mathrm{C}$ do not show a drastic change upon quenching (see images at $t=1952 \mathrm{~s}$ and RT in Figure 5a) as the scattering is predominantly from solid nanowires and nanocrystals. Considering Figure $5 \mathrm{c}$ more closely, one can see that the average width of nanowires gradually increases with time, while the height is relatively time independent. This implies that the incoming droplets feeding the growth of the nanowires add preferentially to the sides of nanowires and not to the tops. This is direct evidence for anisotropic growth, as requirement for nanowire formation.

To summarize, based on real-time GISAXS studies combined with electron microscopy, the growth mechanism of $\mathrm{VO}_{2}$ nanowires is interpreted in terms of supercooled liquid nanodroplets. Specifically, $\mathrm{V}_{2} \mathrm{O}_{5}$ (the lowest melting point vanadium oxide) is vaporized from the source - either by oxidation from background $\mathrm{O}_{2}$ in the rare gas flow or by disproportionation of the $\mathrm{VO}_{2}$ sample that is then transported as vapor to the growth substrate. Here nanometer size $\mathrm{V}_{2} \mathrm{O}_{5}$ droplets form with a freezing point that is strongly suppressed below that of the bulk due to their small size and interactions with the substrate, which does not allow the droplet to fully wet the surface. These $\mathrm{V}_{2} \mathrm{O}_{5}$ nanodroplets then grow by Ostwald ripening or coalescence and the solid phase melting point rises above the substrate temperature. Continued growth of the droplet leads to supercooled $\mathrm{V}_{2} \mathrm{O}_{5}$ that can then crystallize by subsequent loss of $\mathrm{O}_{2}$ in the reducing environment of the furnace forming $\mathrm{VO}_{2}$ crystallites and nanowires. Under mildly oxidizing conditions $\mathrm{V}_{2} \mathrm{O}_{5}$ nanowires form instead. After nanowire formation, nanodroplets continue to feed the growth process.

A remarkable aspect of nanowire growth from supercooled liquid nanodroplets is the sensitivity of the growth to the wetting forces and hence to the characteristics of the substrate. For example, using r-cut sapphire as a substrate, we find epitaxially aligned nanowires whose growth direction is out of the plane of the substrate (Figure 1g). It is also noteworthy that the width distribution for $\mathrm{VO}_{2}$ nanowires grown on r-cut sapphire is narrower and the average width is smaller than for samples grown in plane (compare Figure S5b with Figure S4b). This suggests that the shape of the nanodroplets as determined by wetting forces as well as the possibility for epitaxial growth on this surface are key factors in determining the direction of the nanowire growth.

Figure S6, panels a and b, shows additional examples of how the substrate can be used to influence the growth; namely, how substrate defects can be used to influence the relative positions of several nanowires. Figure S6a shows $\mathrm{VO}_{2}$ nanowires growing (corner-to-corner) preferentially 
along a line defect of the $\mathrm{SiO}_{2} / \mathrm{Si}$ substrate. The substrate defect serves to orient the neighboring nanowires, which grow with opposing corners located on the defect. It is also interesting that the nanowires all exhibit quite similar lengths. Figure S6b shows how a line defect on r-cut sapphire influences the positions at which out-of-plane $\mathrm{VO}_{2}$ nanowires grow. Here, preferential growth along the line defect is seen, suggesting that mobility of liquid nanodroplets along the line defect allows for more rapid delivery of material to some nanowires than to others.

One of the key tests of our understanding of this new mechanism is the extent to which these insights can be applied to other nanowire systems. Furthermore, the simplicity of the concept of the nanowire growth that we have observed from supercooled liquid nanodroplets suggests a certain degree of generality. Table 1 shows bulk melting point data for a number of metal oxides and conditions where nanowires and nanorods have been successfully grown. In all cases, we easily found conditions where nanowires grow by adjusting growth conditions in the temperature range from $50{ }^{\circ} \mathrm{C}$ to $\sim 200{ }^{\circ} \mathrm{C}$ below the melting point of the lowest melting oxide. In most cases, we found that the lowest melting oxide was an appropriate source material, but even higher melting oxides are (in some cases) suitable. In this way in addition to the $\mathrm{VO}_{2}$ and $\mathrm{V}_{2} \mathrm{O}_{5}$ nanowires already shown, we have successfully grown $\mathrm{MoO}_{2}, \mathrm{MoO}_{3}, \mathrm{RuO}_{2}$, and $\mathrm{Fe}_{3} \mathrm{O}_{4}$ single crystal nanowires (Table 1). SEM images of these nanowires can be found in Figure S7.

In conclusion, we report a simple strategy to synthesize a number of highly crystalline metal oxide nanowires via supercooled liquid nanodroplets. We have presented evidence for nanowire growth well below the bulk melting point of $\mathrm{VO}_{2}$, without the use of metal catalyst particles like those used in VLS growth, and shown direct evidence of the presence of ripening supercooled liquid nanodroplets using in situ GISAXS. SEM images also show evidence of Ostwald ripening and a strong sensitivity of the growth to substrate properties. We have extended this concept to the synthesis of a variety of metal oxide nanowire systems, demonstrating the simplicity and generality of this new approach.

Acknowledgment. We gratefully acknowledge the financial support from the Partnership for International Research and Education - for Electronic Chemistry and Catalysis at Interfaces - NSF Grant No. OISE-0530268. The work at Argonne National Laboratory was supported by the US Department of Energy, BES-Chemical Sciences and BESScientific User Facilities under Contract DE-AC-0206CH11357 with UChicago Argonne, LLC, Operator of
Argonne National Laboratory. S.V. gratefully acknowledges the support by the Air Force Office of Scientific Research. We also thank Mr. Kayrat Sabyrov's contribution to this work.

Supporting Information Available: Additional results presented as Figures $\mathrm{S} 1-\mathrm{S} 7$. This material is available free of charge via the Internet at http://pubs.acs.org.

\section{References}

(1) Kolmakov, A.; Moskovits, M. Ann. Rew. Mater. Res. 2004, 34, 151.

(2) Gratzel, M. Nature 2001, 414, 338.

(3) Stern, E.; Klemic, J. F.; Routenberg, D. A.; Wyrembak, P. N.; TurnerEvans, D. B.; Hamilton, A. D.; LaVan, D. A.; Fahmy, T. M.; Reed, M. A. Nature 2007, 445, 519.

(4) Huang, M. H.; Mao, S.; Feick, H.; Yan, H. Q.; Wu, Y. Y.; Kind, H.; Weber, E.; Russo, R.; Yang, P. D. Science 2001, 292, 1897.

(5) Whitney, T. M.; Jiang, J. S.; Searson, P. C.; Chien, C. L. Science 1993, 261, 1316.

(6) Pan, Z. W.; Dai, Z. R.; Wang, Z. L. Science 2001, 291, 1947.

(7) Persson, A. I.; Larsson, M. W.; Stenstrom, S.; Ohlsson, B. J.; Samuelson, L.; Wallenberg, L. R. Nat. Mater. 2004, 3, 677.

(8) Wagner, R. S.; Ellis, W. C. Appl. Phys. Lett. 1964, 4, 89.

(9) Morales, A. M.; Lieber, C. M. Science 1998, 279, 208.

(10) Kodambaka, S.; Tersoff, J.; Reuter, M. C.; Ross, F. M. Science 2007, 316, 729.

(11) Hofmann, S.; Sharma, R.; Wirth, C. T.; Cervantes-Sodi, F.; Ducati, C.; Kasama, T.; Dunin-Borkowski, R. E.; Drucker, J.; Bennett, P.; Robertson, J. Nat. Mater. 2008, 7, 372.

(12) Kodambaka, S.; Hannon, J. B.; Tromp, R. M.; Ross, F. M. Nano Lett. 2006, 6, 1292.

(13) Buffat, P.; Borel, J. P. Phys. Rev. A 1976, 13, 2287.

(14) Peters, K. F.; Chung, Y. W.; Cohen, J. B. Appl. Phys. Lett. 1997, 71, 2391.

(15) Baletto, F.; Ferrando, R. Rev. Mod. Phys. 2005, 77, 371.

(16) Peters, K. F.; Cohen, J. B.; Chung, Y. W. Phys. Rev. B 1998, 57, 13430.

(17) Madras, G.; McCoy, B. J. J. Chem. Phys. 2002, 117, 8042.

(18) Wu, Y. Y.; Yang, P. D. J. Am. Chem. Soc. 2001, 123, 3165.

(19) Sutter, E.; Sutter, P. Nano Lett. 2008, 8, 411.

(20) Renaud, G.; Lazzari, R.; Revenant, C.; Barbier, A.; Noblet, M.; Ulrich, O.; Leroy, F.; Jupille, J.; Borensztein, Y.; Henry, C. R.; Deville, J. P.; Scheurer, F.; Mane- Mane, J.; Fruchart, O. Science 2003, 300, 1416.

(21) Lee, B.; Seifert, S.; Riley, S. J.; Tikhonov, G.; Tomczyk, N. A.; Vajda, S.; Winans, R. E. J. Chem. Phys. 2005, 123, 074701.

(22) Leroy, F.; Renaud, G.; Letoublon, A.; Lazzari, R.; Mottet, C.; Goniakowski, J. Phys. Rev. Lett. 2005, 95, 185501.

(23) Lee, S.; Molina, L. M.; Lopez, M. J.; Alonso, J. A.; Hammer, B.; Lee, B.; Seifert, S.; Winans, R. E.; Elam, J. W.; Pellin, M. J.; Vajda, S. Angew. Chem., Int. Ed. 2009, 48, 1467.

(24) Baik, J. M.; Kim, M. H.; Larson, C.; Wodtke, A. M.; Moskovits, M. J. Phys. Chem. C 2008, 112, 13328.

(25) Guiton, B. S.; Gu, Q.; Prieto, A. L.; Gudiksen, M. S.; Park, H. J. Am. Chem. Soc. 2005, 127, 498.

(26) Sohn, J. I.; Joo, H. J.; Porter, A. E.; Choi, C. J.; Kim, K.; Kang, D. J.; Welland, M. E. Nano Lett. 2007, 7, 1570.

(27) Piccirillo, C.; Binions, R.; Parkin, I. P. Chem. Vapor Depos. 2007, $13,145$.

(28) Sanchez, C.; Livage, J.; Lucazeau, G. J. Raman Spectrosc. 1982, 12, 68.

NL902357Q 